\title{
The Telegraph Equation and Its Solution by Reduced Differential Transform Method
}

\author{
Vineet K. Srivastava, ${ }^{1}$ Mukesh K. Awasthi, ${ }^{2}$ R. K. Chaurasia, ${ }^{3}$ and M. Tamsir ${ }^{4}$ \\ ${ }^{1}$ ISRO Telemetry, Tracking and Command Network (ISTRAC), Bangalore 560058, India \\ ${ }^{2}$ Department of Mathematics, University of Petroleum and Energy Studies, Dehradun 248007, India \\ ${ }^{3}$ The ICFAI University, Jaipur 302031, India \\ ${ }^{4}$ Department of Mathematics, Graphic Era University, Dehradun 248002, India
}

Correspondence should be addressed to Vineet K. Srivastava; vineetsriiitm@gmail.com

Received 18 May 2013; Revised 8 July 2013; Accepted 7 August 2013

Academic Editor: Ahmed Rachid

Copyright (c) 2013 Vineet K. Srivastava et al. This is an open access article distributed under the Creative Commons Attribution License, which permits unrestricted use, distribution, and reproduction in any medium, provided the original work is properly cited.

One-dimensional second-order hyperbolic telegraph equation was formulated using Ohm's law and solved by a recent and reliable semianalytic method, namely, the reduced differential transform method (RDTM). Using this method, it is possible to find the exact solution or a closed approximate solution of a differential equation. Three numerical examples have been carried out in order to check the effectiveness, the accuracy, and convergence of the method. The RDTM is a powerful mathematical technique for solving wide range of problems arising in science and engineering fields.

\section{Introduction}

In this modern era communication system plays a key role in the worldwide society. High frequency communication systems continue to benefit from significant industrial attention, triggered by a host of radio frequency (RF) and microwave communication (MW) systems. These systems use the transmission media for transferring the information carrying signal from one point to another point. This transmission media can be categorized into two groups, namely, guided and unguided. In guided medium the signal is transferred through the coaxial cable or transmission line. These guided media are capable of transporting the high frequency voltage and current waves. While in unguided media electromagnetic waves carry the signal over part of or the entire communication path through RF and MW channels. These electromagnetic waves are transmitted and received through antenna. In guided transmission media, specifically cable transmission medium is investigated to address the problem of efficient telegraphic transmission. A cable transmission medium can be classified as a guided transmission medium and represents a physical system that directly propagates the information between two or more locations. In order to optimize the guided communication system it is necessary to determine or project power and signal losses in the system, because all the systems have such losses. To determine these losses and eventually ensure a maximum output, it is necessary to formulate some kind of equation with which to calculate these losses.

In this paper a mathematical derivation for the telegraph equation in terms of voltage and current for a section of a transmission line has been formulated and the obtained mathematical equation is solved by a very recent approximate analytical method, namely, the reduced differential transform method (RDTM).

Let us consider an infinitesimal piece of telegraph cable wire as an electrical circuit shown in Figure 1. Further assume that the cable is imperfectly insulated so that there are both capacitance and current leakage to ground.

Suppose $x$ is the distance from sending end of the cable; $u(x, t)$ is the voltage at any point and any time, on the cable; $i(x, t)$ is the current at any point and any time, on the cable; $R$ denotes the resistance of the cable; $C$ denotes the capacitance to the ground; $L$ denotes the inductance of the cable; $G$ denotes the conductance to the ground. 


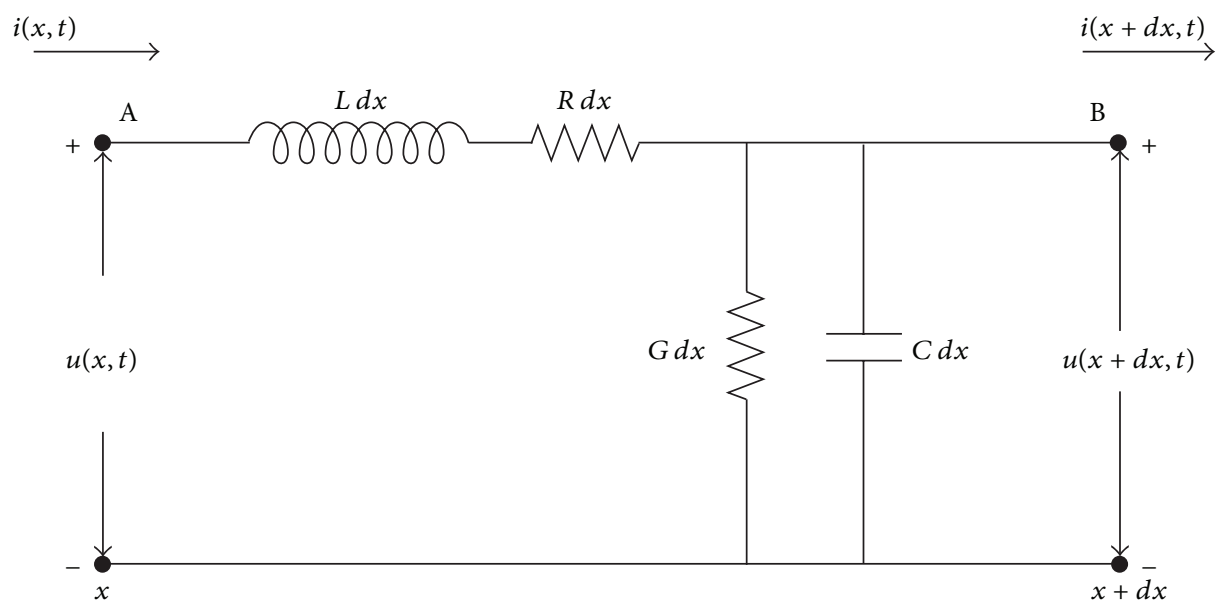

FIGURE 1: Schematic diagram of telegraphic transmission line with leakage. by

Then by Ohm's law, the voltage across the resistor is given

$$
u=i R
$$

Further, the voltage drop across the inductor is given as

$$
u=L \frac{d i}{d t}
$$

The voltage drop across the capacitor is given by

$$
u=\frac{1}{C} \int i d t
$$

The voltage at terminal $\mathrm{B}$ is equal to the voltage at terminal $\mathrm{A}$, minus the drop in voltage along the element $A B$, so if (1), (2), and (3) are combined together, then we have

$$
u(x+d x, t)-u(x, t)=-[R d x] i-[L d x] \frac{\partial i}{\partial t} .
$$

Let $d x \rightarrow 0$, and differentiating (4) partially with respect to $x$, we have

$$
\frac{\partial u}{\partial x}=-R i-L \frac{\partial i}{\partial t}
$$

Similarly, the current at terminal B is equal to the current at terminal A minus the current through leakage to the ground, so that we get

$$
i(x+d x, t)=i(x, t)-[G d x] u-i_{C} d x .
$$

The current through the capacitor is given as

$$
i_{C}=C \frac{\partial u}{\partial t}
$$

Differentiating (4) with respect to $t$ and (7) with respect to $x$ and eliminating the derivatives of $u$, we have [1]

$$
c^{2} \frac{\partial^{2} i}{\partial x^{2}}=\frac{\partial^{2} i}{\partial t^{2}}+(\alpha+\beta) \frac{\partial i}{\partial t}+(\alpha \beta) i
$$

Similarly, we have

$$
c^{2} \frac{\partial^{2} u}{\partial x^{2}}=\frac{\partial^{2} u}{\partial t^{2}}+(\alpha+\beta) \frac{\partial u}{\partial t}+(\alpha \beta) u,
$$

where

$$
\alpha=\frac{G}{C}, \quad \beta=\frac{R}{L}, \quad c^{2}=\frac{1}{L C} .
$$

Equations (8) and (9) are known as one-dimensional hyperbolic second-order telegraph equations.

\section{Reduced Differential Transform Method (RDTM)}

In this section, we introduce the basic definitions of the reduced differential transformations.

Consider a function of two variables $w(x, t)$ and assume that it can be represented as a product of two singlevariable functions; that is, $w(x, t)=F(x) G(t)$. On the basis of the properties of the one-dimensional differential transformation, the function $w(x, t)$ can be represented as [2]

$$
w(x, t)=\sum_{i=0}^{\infty} F(i) x^{i} \sum_{j=0}^{\infty} G(j) t^{j}=\sum_{i=0}^{\infty} \sum_{j=0}^{\infty} W(i, j) x^{i} t^{j},
$$

where $W(i, j)=F(i) G(j)$ is called the spectrum of $w(x, t)$.

Let $R_{D}$ denote the reduced differential transform operator and $R_{D}^{-1}$ the inverse reduced differential transform operator. The basic definition and operation of the RDTM are introduced below.

Definition 1. If $w(x, t)$ is analytic and continuously differentiable with respect to space variable $x$ and time variable $t$ in the domain of interest, then the spectrum function [3-5]

$$
R_{D}[w(x, t)] \approx W_{k}(x)=\frac{1}{k !}\left[\frac{\partial^{k}}{\partial t^{k}} w(x, t)\right]_{t=t_{0}}
$$

is the reduced transformed function of $w(x, t)$. 
In this paper, (lowercase) $w(x, t)$ represents the original function while (uppercase) $W_{k}(x)$ stands for the reduced transformed function. The differential inverse reduced transform of $W_{k}(x)$ is defined as [3-5]

$$
R_{D}^{-1}\left[W_{k}(x)\right] \approx w(x, t)=\sum_{k=0}^{\infty} W_{k}(x)\left(t-t_{0}\right)^{k} .
$$

Combining (12) and (13), we get

$$
w(x, t)=\sum_{k=0}^{\infty} \frac{1}{k !}\left[\frac{\partial^{k}}{\partial t^{k}} w(x, t)\right]_{t=t_{0}}\left(t-t_{0}\right)^{k} .
$$

From (14), it can be seen that the concept of the reduced differential transform is derived from the power series expansion of the function.

Definition 2. If $u(x, t)=R_{D}^{-1}\left[U_{k}(x)\right], v(x, t)=R_{D}^{-1}\left[V_{k}(x)\right]$, and the convolution $\otimes$ denotes the reduced differential transform version of the multiplication, then the fundamental operations of the reduced differential transform are shown in Table 1.

\section{Computational Illustrations}

In this section, we describe the method explained in Section 2 taking three examples of both linear and nonlinear telegraph equations to validate the efficiency and reliability of the RDTM.

Example 1. Consider the linear telegraph equation

$$
\frac{\partial^{2} u}{\partial x^{2}}=\frac{\partial^{2} u}{\partial t^{2}}+2 \frac{\partial u}{\partial t}+u
$$

subject to the initial conditions

$$
\begin{gathered}
u(x, 0)=e^{x}, \\
u_{t}(x, 0)=-2 e^{x} .
\end{gathered}
$$

Applying the RDTM to (15), we obtain the following recurrence relation:

$$
\begin{gathered}
(k+1)(k+2) U_{k+2}(x)+2(k+1) U_{k+1}(x) \\
=\frac{\partial^{2}}{\partial x^{2}} U_{k}(x)-U_{k}(x) .
\end{gathered}
$$

Applying the RDTM to the initial conditions (16), we have

$$
U_{0}(x)=e^{x} ; \quad U_{1}(x)=-2 e^{x} .
$$

\begin{tabular}{|c|c|}
\hline Original function & Reduced differential transformed function \\
\hline$R_{D}[u(x, t) v(x, t)]$ & $U_{k}(x) \otimes V_{k}(x)=\sum_{r=0}^{k} U_{r}(x) V_{k-r}(x)$ \\
\hline$R_{D}[\alpha u(x, t) \pm \beta v(x, t)]$ & $\alpha U_{k}(x) \pm \beta V_{k}(x)$ \\
\hline$R_{D}\left[\frac{\partial}{\partial x} u(x, t)\right]$ & $\frac{\partial}{\partial x} U_{k}(x)$ \\
\hline$R_{D}\left[\frac{\partial}{\partial t} u(x, t)\right]$ & $(k+1) U_{k+1}(x)$ \\
\hline$R_{D}\left[\frac{\partial^{r+s}}{\partial x^{r} \partial t^{s}} u(x, t)\right]$ & $\frac{(k+s) !}{k !} \frac{\partial^{r}}{\partial x^{r}} U_{k+s}(x)$ \\
\hline$R_{D}\left[x^{m} t^{n}\right]$ & $\begin{cases}x^{m}, & k=n \\
0, & \text { otherwise }\end{cases}$ \\
\hline$R_{D}\left[e^{\lambda t}\right]$ & $\frac{\lambda^{k}}{k !}$ \\
\hline$R_{D}[\sin (w t+\alpha)]$ & $\frac{w^{k}}{k !} \sin \left(\frac{\pi k}{2 !}+\alpha\right)$ \\
\hline$R_{D}[\cos (w t+\alpha)]$ & $\frac{w^{k}}{k !} \cos \left(\frac{\pi k}{2 !}+\alpha\right)$ \\
\hline
\end{tabular}

From (18) into (17), we get the following $U_{k}(x)$ values successively:

$$
\begin{gathered}
U_{2}(x)=2 e^{x}=\frac{(-2)^{2}}{2 !} e^{x}, \quad U_{3}(x)=-\frac{4}{3} e^{x}=\frac{(-2)^{3}}{3 !} e^{x}, \\
U_{4}(x)=\frac{2}{3} e^{x}=\frac{(-2)^{4}}{4 !} e^{x}, \ldots, U_{k}(x)=\frac{(-2)^{k}}{k !} e^{x} .
\end{gathered}
$$

TABLE 1: Fundamental operations of the reduced differential transform method.

Using the differential inverse reduced transform of $U_{k}(x)$, we get

$$
\begin{aligned}
u(x, t)= & \sum_{k=0}^{\infty} U_{k}(x) t^{k}=U_{0}(x)+U_{1}(x) t+U_{2}(x) t^{2} \\
& +U_{3}(x) t^{3}+\cdots \\
= & e^{x}\left(1+(-2) t+\frac{(-2)^{2}}{2 !} t^{2}+\frac{(-2)^{3}}{3 !} t^{3}\right. \\
& \left.+\cdots+\frac{(-2)^{k}}{k !} t^{k}+\cdots\right) .
\end{aligned}
$$

The solution (20), in closed form, is given as

$$
u(x, t)=e^{x-2 t} .
$$

Example 2. Consider the linear telegraph equation

$$
\frac{\partial^{2} u}{\partial x^{2}}=\frac{\partial^{2} u}{\partial t^{2}}+4 \frac{\partial u}{\partial t}+4 u
$$

with the initial conditions

$$
\begin{gathered}
u(x, 0)=1+e^{2 x}, \\
u_{t}(x, 0)=-2 .
\end{gathered}
$$

Operating the RDTM to (23), we obtain the recurrence equation

$$
\begin{gathered}
(k+1)(k+2) U_{k+2}(x)+4(k+1) U_{k+1}(x) \\
=\frac{\partial^{2}}{\partial x^{2}} U_{k}(x)-4 U_{k}(x) .
\end{gathered}
$$




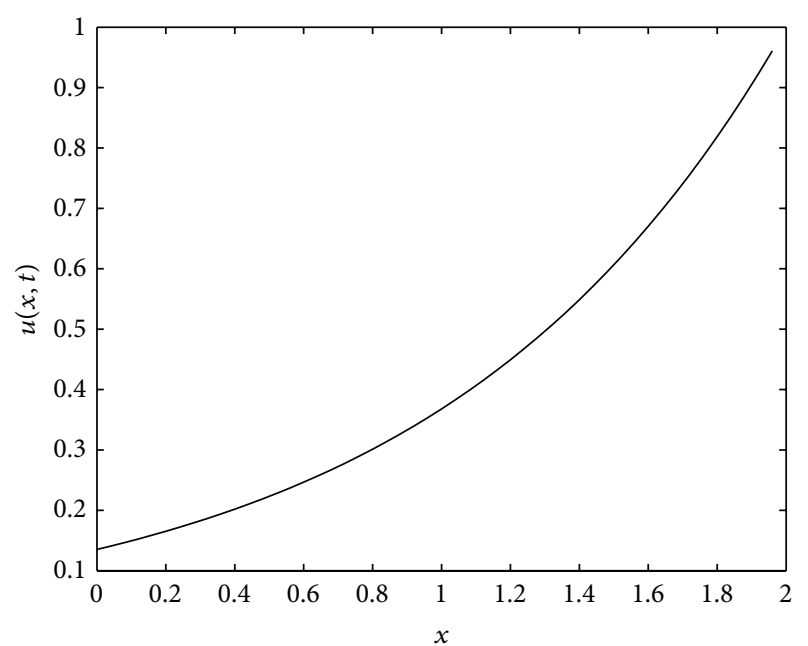

FIGURE 2: Plot of $u(x, t)$ with respect to $x, x \in[0,2]$ at $t=1$ for Examples 1 and 3.

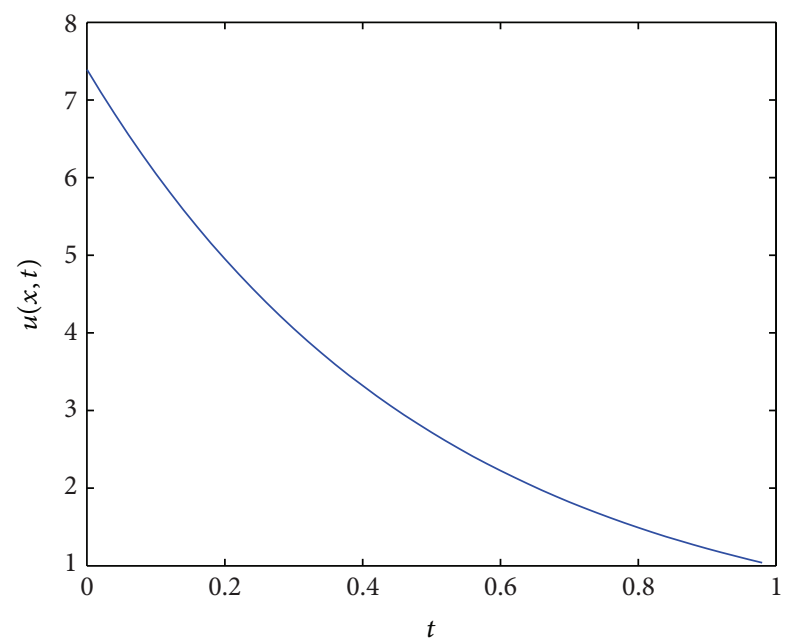

FIgURE 3: Plot of $u(x, t)$ with respect to $t, t \in[0,1]$ at $x=2$ for Examples 1 and 3.

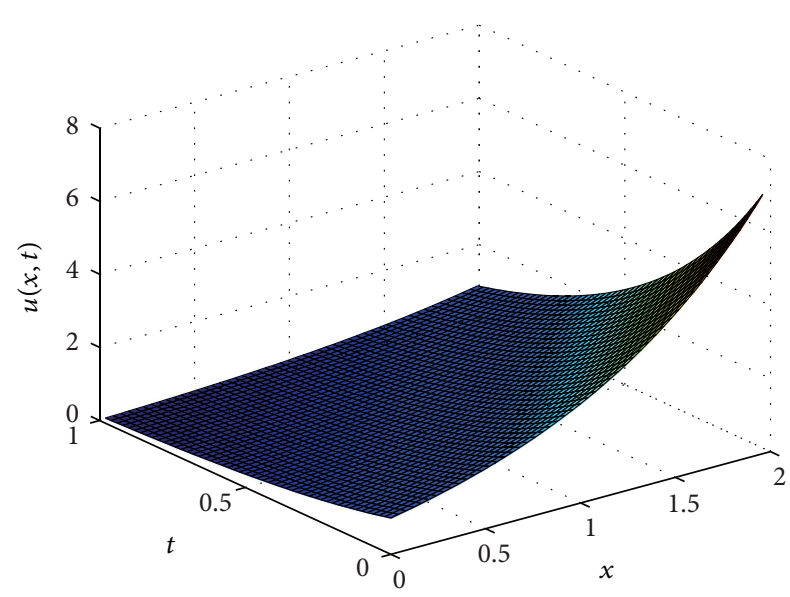

FIGURE 4: Depiction of solution $u(x, t)$ in the domain $x \in[0,2]$ and $t \in[0,1]$ for Examples 1 and 3 .

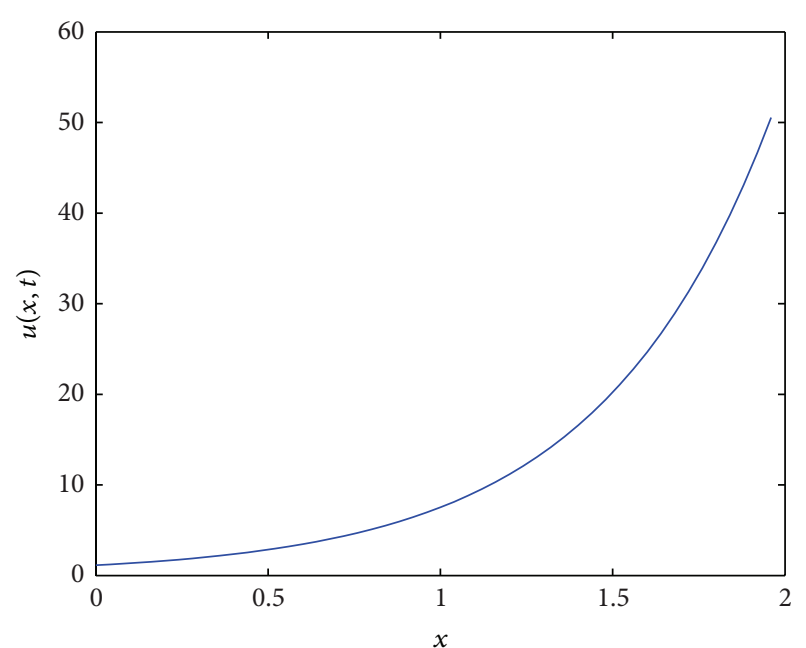

Figure 5: Plot of $u(x, t)$ with respect to $x, x \in[0,2]$ at $t=1$ for Example 2.

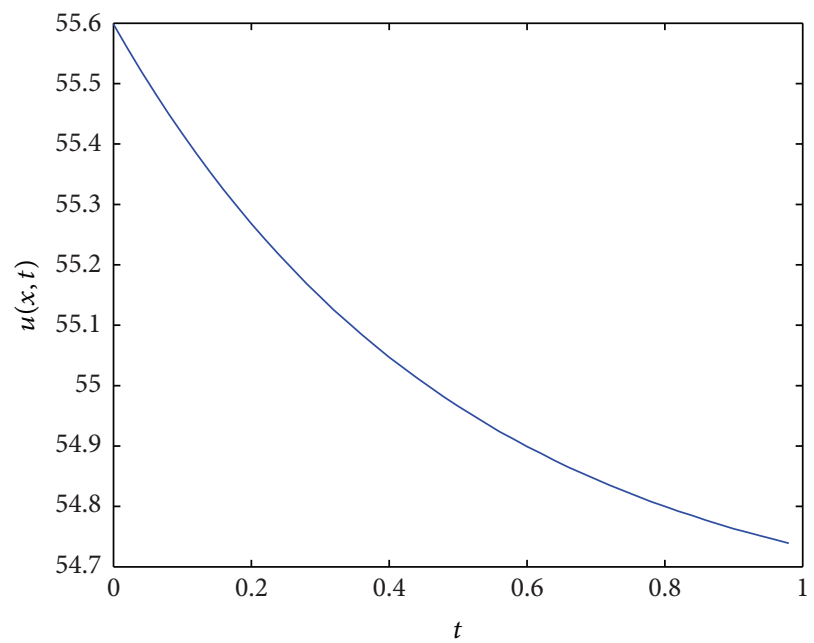

FIgURE 6: Plot of $u(x, t)$ with respect to $t, t \in[0,1]$ at $x=2$ for Example 2.

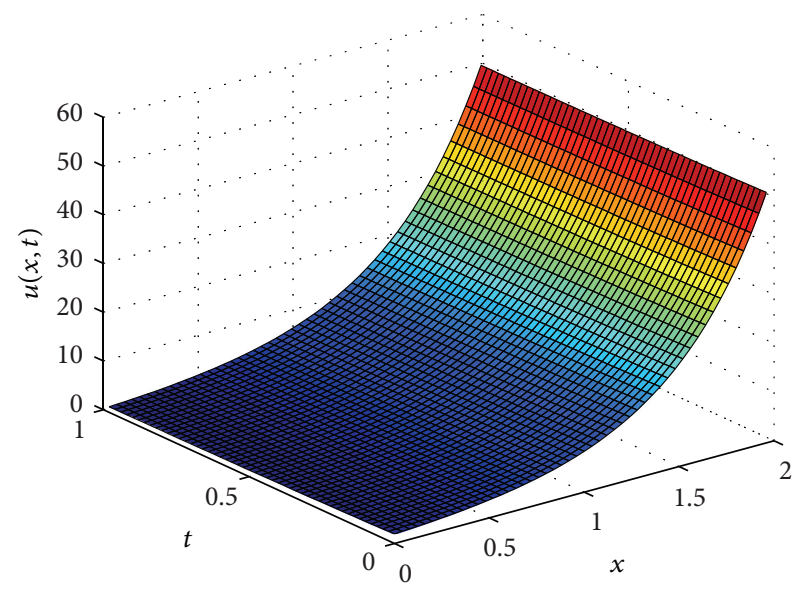

FIGURE 7: Depiction of solution $u(x, t)$ in the domain $x \in[0,2]$ and $t \in[0,1]$ for Example 2 . 
Applying the RDTM to the initial conditions (24), we get

$$
U_{0}(x)=1+e^{2 x} ; \quad U_{1}(x)=-2 .
$$

Applying (25) to (24) we get the following $U_{k}(x)$ values successively:

$$
\begin{gathered}
U_{2}(x)=2=\frac{(-2)^{2}}{2 !} ; \quad U_{3}(x)=-\frac{4}{3}=\frac{(-2)^{3}}{3 !} ; \\
U_{4}(x)=\frac{2}{3}=\frac{(-2)^{4}}{4 !}, \ldots, U_{k}(x)=\frac{(-2)^{k}}{k !} .
\end{gathered}
$$

Using the differential inverse reduced transform of $U_{k}(x)$, we get

$$
\begin{aligned}
u(x, t)= & \sum_{k=0}^{\infty} U_{k}(x) t^{k}=U_{0}(x)+U_{1}(x) t+U_{2}(x) t^{2} \\
& +U_{3}(x) t^{3}+\cdots \\
= & e^{2 x}+\left[1+(-2) t+\frac{(-2)^{2}}{2 !} t^{2}+\frac{(-2)^{3}}{3 !} t^{3}\right. \\
& \left.+\cdots+\frac{(-2)^{k}}{k !} t^{k}+\cdots\right]
\end{aligned}
$$

The exact solution, in closed form, is given as

$$
u(x, t)=e^{2 x}+e^{-2 t} \text {. }
$$

Example 3. Consider the following nonlinear telegraph equation:

$$
\frac{\partial^{2} u}{\partial x^{2}}=\frac{\partial^{2} u}{\partial t^{2}}+2 \frac{\partial u}{\partial t}+u^{2}-e^{2 x-4 t}+e^{x-2 t}
$$

under the initial conditions

$$
\begin{gathered}
u(x, 0)=e^{x}, \\
u_{t}(x, 0)=-2 e^{x} .
\end{gathered}
$$

Applying the RDTM technique to (29), we obtain the following iterative formula:

$$
\begin{gathered}
(k+1)(k+2) U_{k+2}(x)+2(k+1) U_{k+1}(x) \\
=\frac{\partial^{2}}{\partial x^{2}} U_{k}(x)-\sum_{r=0}^{k} U_{r}(x) U_{k-r}(x) \\
+e^{2 x}\left(\frac{(-4)^{k}}{k !}\right)-e^{x}\left(\frac{(-2)^{k}}{k !}\right) .
\end{gathered}
$$

Applying the RDTM to the initial conditions (30), we get

$$
U_{0}(x)=e^{x} ; \quad U_{1}(x)=-2 e^{x} .
$$

Using (32) in (31), we get the following $U_{k}(x)$ values successively:

$$
\begin{gathered}
U_{2}(x)=2 e^{x}=\frac{(-2)^{2}}{2 !} e^{x} ; \quad U_{3}(x)=-\frac{4}{3} e^{x}=\frac{(-2)^{3}}{3 !} e^{x} ; \\
U_{4}(x)=\frac{2}{3} e^{x}=\frac{(-2)^{4}}{4 !} e^{x}, \ldots, U_{k}(x)=\frac{(-2)^{k}}{k !} e^{x} .
\end{gathered}
$$

Using the differential inverse reduced transform of $U_{k}(x)$, we get

$$
\begin{aligned}
u(x, t)= & \sum_{k=0}^{\infty} U_{k}(x) t^{k}=U_{0}(x)+U_{1}(x) t+U_{2}(x) t^{2} \\
& +U_{3}(x) t^{3}+\cdots \\
= & e^{x}\left(1+(-2) t+\frac{(-2)^{2}}{2 !} t^{2}+\frac{(-2)^{3}}{3 !} t^{3}\right. \\
& \left.+\cdots+\frac{(-2)^{k}}{k !} t^{k}+\cdots\right) .
\end{aligned}
$$

The solution (34), in closed form, is given as

$$
u(x, t)=e^{x-2 t}
$$

Here, we have also presented the numerical results through various graphs. The variation of voltage $u(x, t)$ with distance $x$ at a particular time $t$ has been drawn in Figure 2 while the voltage $u(x, t)$ with time $t$ at a particular distance $x$ is shown in Figure 3 for Example 1. We notice that the voltage grows exponentially with distance at any instant of time while at a particular distance, there is an exponential decay in $u(x, t)$. It may also be seen from (21) that if we take $t$ as a constant, $u(x, t)=c e^{x}$ while at constant distance $u(x, t)$ will be constant multiple of $e^{-2 t}$. The three-dimensional variation of the solution $u(x, t)$ versus $x$ and $t$ is depicted in Figure 4 .

Figure 5 shows that the voltage $u(x, t)$ has been drawn at a particular time $t$ for Example 2. It is observed that the voltage $u(x, t)$ increases exponentially if $t$ is constant. Figure 6 shows that at a particular distance $x$, there is an exponential decrease in the voltage $u(x, t)$ for Example 2. The same thing is observed from the analytical solution (28). Figure 7 depicts the three-dimensional variation of $u(x, t)$ versus $x$ and $t$. The analytical expression for Example 3 is the same as the one obtained for Example 1 and therefore, the numerical results will also be the same.

\section{Conclusions}

In this work, the reduced differential transform method has been implemented for one-dimensional second-order hyperbolic linear and nonlinear telegraph equations. The method is applied in a direct way without using linearization, transformation, discretization, or restrictive assumptions. The results show that the introduced technique is highly accurate, rapidly converge, and very easy to apply in the various engineering problems.

\section{References}

[1] R. J. Schwarz and B. Friedland, Linear Systems, McGraw-Hill, New York, NY, USA, 1965.

[2] R. Abazari and M. Ganji, "Extended two-dimensional DTM and its application on nonlinear PDEs with proportional delay," International Journal of Computer Mathematics, vol. 88, no. 8, pp. 1749-1762, 2011. 
[3] Y. Keskin and G. Oturanç, "Reduced differential transform method for partial differential equations," International Journal of Nonlinear Sciences and Numerical Simulation, vol. 10, no. 6, pp. 741-749, 2009.

[4] Y. Keskin and G. Oturanc, "Reduced differential transform method for solving linear and nonlinear wave equations," Iranian Journal of Science and Technology A, vol. 34, no. 2, pp. 113-122, 2010.

[5] R. Abazari and M. Abazari, "Numerical simulation of generalized Hirota-Satsuma coupled KdV equation by RDTM and comparison with DTM," Communications in Nonlinear Science and Numerical Simulation, vol. 17, no. 2, pp. 619-629, 2012. 

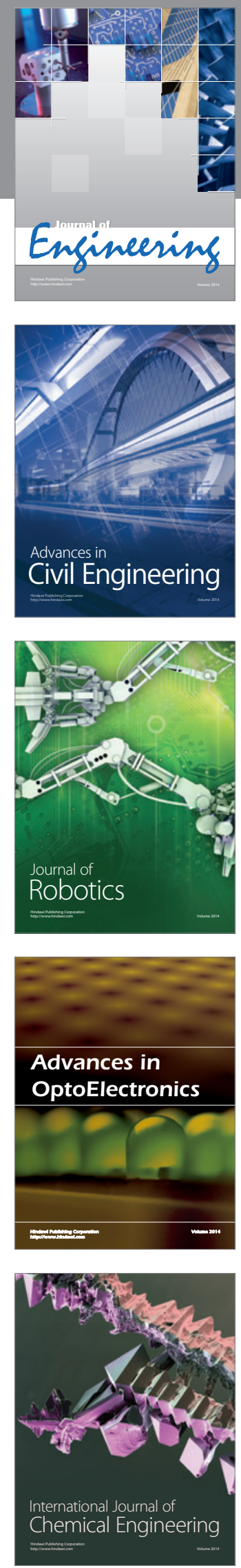

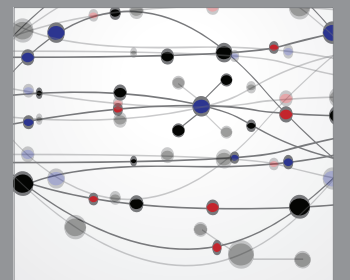

The Scientific World Journal
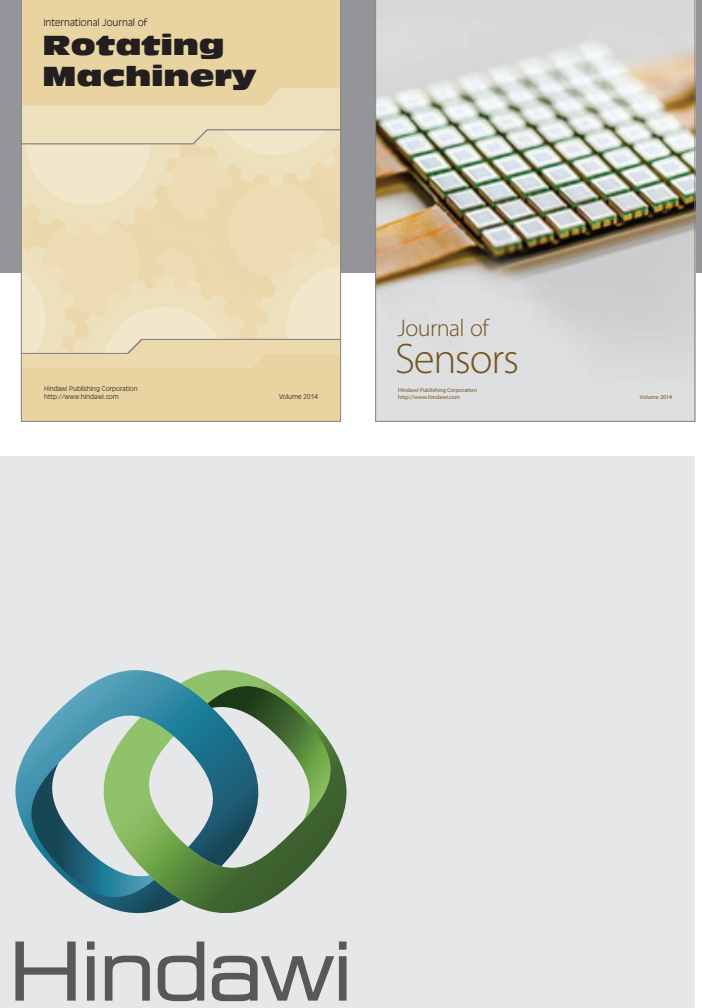

Submit your manuscripts at http://www.hindawi.com
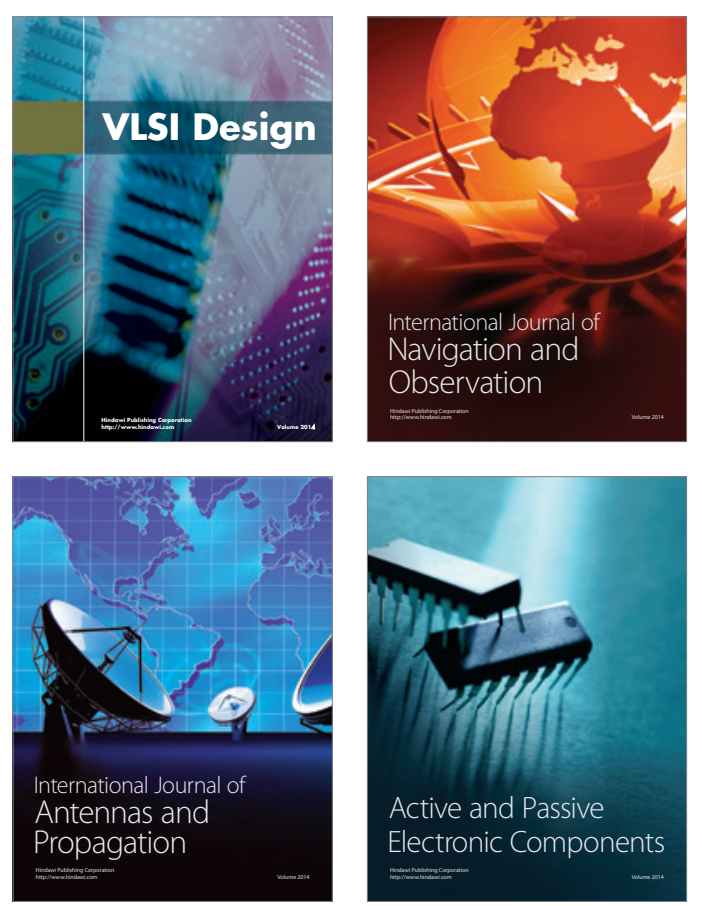
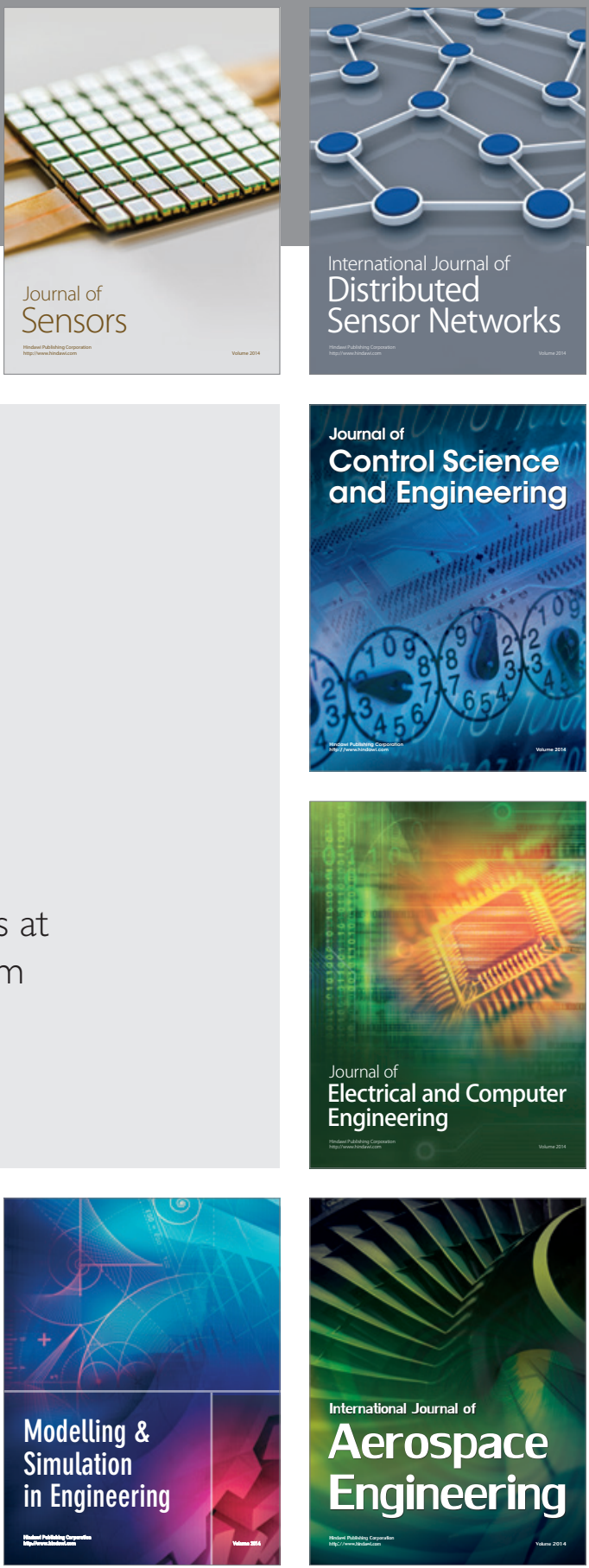

Journal of

Control Science

and Engineering
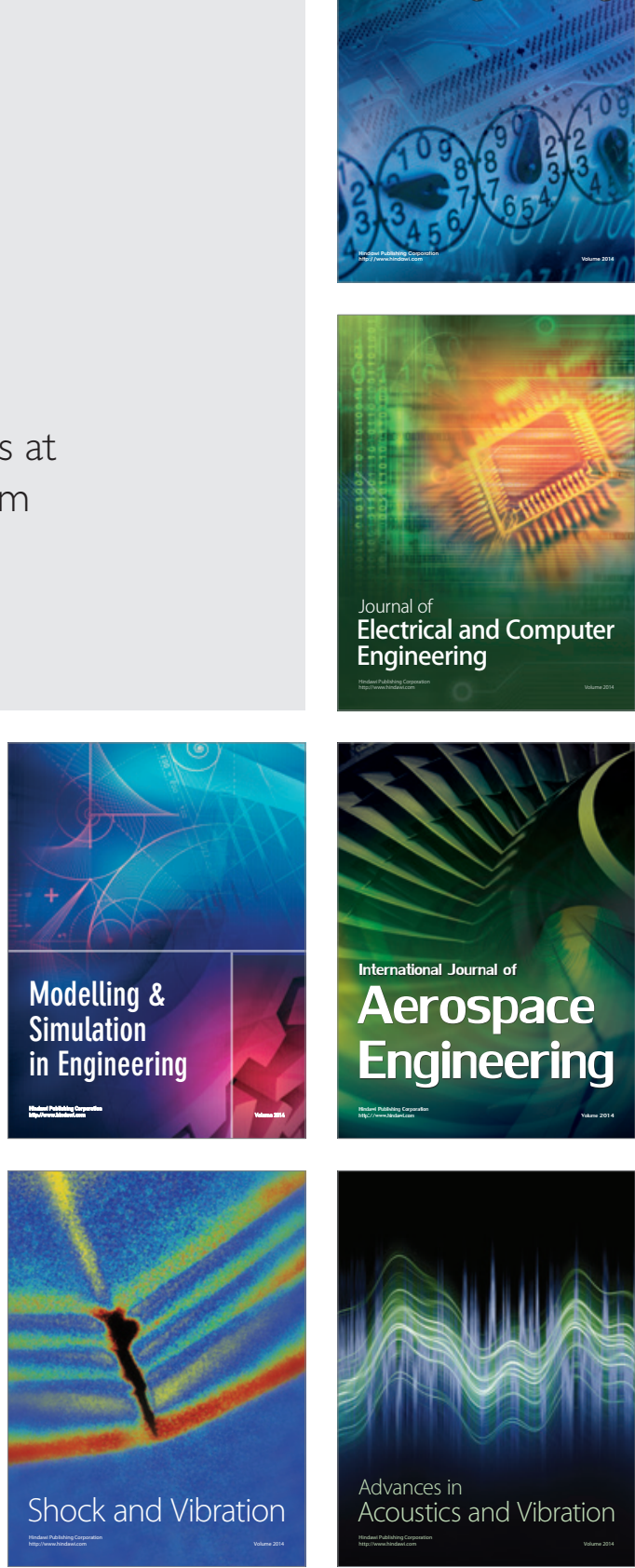